\title{
Ammonium Chlorochromate Adsorbed on Alumina for Cleavage of Semicarbazones and Phenylhydrazones under Classical Heating and Microwave Irradiation in Solvent-free System
}

\author{
Majid M. Heravi, ${ }^{*, a, b}$ Ali J. Sabaghian, ${ }^{b}$ Khadijeh Bakhtiari ${ }^{a}$ and Mitra Ghassemzadeh ${ }^{c}$ \\ ${ }^{a}$ Department of Chemistry, School of Sciences, Azzahra University, Vanak, Tehran, Iran \\ ${ }^{b}$ Department of Chemistry, School of Sciences, Ferdowsi University of Mashhad, Mashhad, Iran \\ ${ }^{c}$ Chemistry \& Chemical Engineering Research Center of Iran, Tehran, Iran
}

\begin{abstract}
Clorocromato de amônio suportado em alumina, regenera eficientemente compostos carbonílicos de suas semicarbazonas e fenilidrazonas correspondentes, sob aquecimento clássico em condições não-aquosas e irradiação de microondas em sistema livre de solvente.

Ammonium chlorochromate supported onto alumina efficiently regenerates carbonyl compounds from their corresponding semicarbazones and phenylhydrazones under classical heating in non-aqueous conditions and microwave irradiation in solvent-free system.
\end{abstract}

Keywords: semicarbazones, phenylhydrazones, deprotection, ammonium chlorochromate, solvent-free

\section{Introduction}

Semicarbazones and phenylhydrazones serve as important synthetic intermediates and can be preferably used for isolation, purification, characterization and protection of aldehydes and ketones. ${ }^{1,2}$ Because of these significances a plethora of reagents and methods have been reported for desemicarbazonization ${ }^{3}$ and oxidative cleavage of phenylhydrazones. ${ }^{4}$

Ammonium chlorochromate has been employed as a versatile reagent in organic synthesis. ${ }^{5}$ Reagents impregnated on several solid supports have gained popularity in organic synthesis because of their selectivity and ease of manipulation. ${ }^{6}$ Microwave enhanced chemical reactions, in general ${ }^{7}$ and especially on inorganic solid supports and under solvent-free conditions have attracted attention recently. ${ }^{8}$ They offer several advantages over conventional homogeneous and heterogeneous reactions with respect to high reaction rates and yields. ${ }^{9}$

\section{Results and Discussion}

In continuation of our investigations of organic reactions using supported reagents ${ }^{10}$ and particularly under microwave irradiation in solvent-free system, ${ }^{11}$ we now

* e-mail: mmh1331@yahoo.com report a facile and selective oxidative cleavage of semicarbazones and phenylhydrazones using alumina supported ammonium chlorochromate under classical heating and microwave assisted conditions (Scheme 1).

The reagent is easily prepared by addition of weighed amount of alumina to a solution of ammonium chlorochromate in water and rotary evaporating to dryness. In the case of classical heating conditions, among the solvents tested for this reaction $\left(\mathrm{CHCl}_{3}, \mathrm{CH}_{2} \mathrm{Cl}_{2}\right.$, THF, toluene), THF and toluene were found to be the most efficient for maximum yield of the products. The oxidative cleavage is conducted by heating of an appropriate either semicarbazone or phenylhydrazone with the supported reagent in THF and toluene respectively.

We observed that in the absence of alumina, the reactions are slow at reflux temperature and even for an extended period of time unconverted starting materials were recovered and more seriously, the molten reagent is adhered to the walls of vessel. However, when ammonium chlorochromate was supported onto alumina cleavage of carbon-nitrogen double bonds in semicarbazones and phenylhydrazones were occurred efficiently, in high yields.

During the course of our investigation on organic manipulation in solvent free systems, ${ }^{10,11}$ we have observed a relatively useful microwave effect. The salient features of this MW approach are improved reaction times and cleaner reactions. 
Among the various solid supports examined such as montmorillonite K-10, kaolin, silica gel and alumina, the latter proved to be superior in terms of yield and reaction time.

The reaction under microwave irradiation was simply conducted by mixing of an appropriate either neat semicarbazone or phenylhydrazone using pestle and mortar and placing the resultant mixture in a beaker. The beaker was then placed in a household microwave oven and irradiated. The reactions are completed in the indicated times (Table 1).

The reactions are relatively clean. No tar formation was observed and most importantly no over oxidation to carboxylic acid was occurred. It is noteworthy to mention that this reagent is not suitable for aliphatic carbonyl compounds.

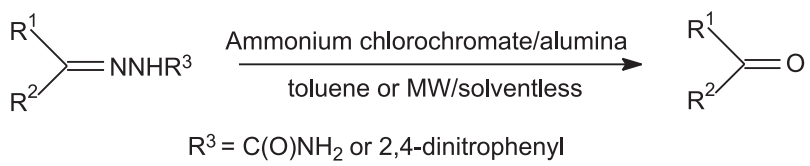

Scheme 1.

\section{Conclusions}

In conclusion an environmentally benign and safe oxidant on a solid support has been introduced. Rapid reactions, high yields and the use of an inexpensive and non-corrosive reagent under microwave irradiation in solvent-free system are attractive features of this protocol.

\section{Experimental}

All products were identified by comparison of their physical and spectroscopic data with those of authentic samples. Semicarbazones and 2,4-dinitrophenylhydrazones were prepared by reaction of aldehydes and ketones with semicarbazide hydrochloride and 2,4-dinitrophenylhydrazine respectively and identified by their melting points and IR spectra. Domestic microwave oven MB-310 Butane was used for the all reactions.

\section{Preparation of ammonium chlorochromate/alumina}

To a solution of chromium trioxide ( $40 \mathrm{~g}, 0.4 \mathrm{~mol})$ in water $(100 \mathrm{~mL})$ ammonium chloride $(21.4 \mathrm{~g}, 0.4 \mathrm{~mol})$ within $15 \mathrm{~min}$ at $40{ }^{\circ} \mathrm{C}$ was added. The mixture was cooled until a yellow-orange solid formed. Reheating to $40{ }^{\circ} \mathrm{C}$ gave a solution. Alumina ( $200 \mathrm{~g}$ ) was then added with stirring at $40{ }^{\circ} \mathrm{C}$. After evaporation of solvent in a rotary evaporator, the orange solid was dried under vacuum for $2 \mathrm{~h}$ at $70{ }^{\circ} \mathrm{C}$. The catalyst thus prepared can be kept for several months in air at room temperature, without losing its activity.

\section{Cleavage of carbon-nitrogen double bond under classical heating. General procedure}

To a solution of an appropriate either semicarbazone or 2,4-dinitrophenylhydrazone ( $2 \mathrm{mmol})$ in either THF or

Table 1. Oxidative cleavage of semicarbazones and phenylhydrazones, using ammonium chlorochromate/alumina under different conditions

\begin{tabular}{|c|c|c|c|c|c|c|c|}
\hline \multirow[t]{2}{*}{ Entry } & \multirow[t]{2}{*}{$\mathrm{R}^{1}$} & \multirow[t]{2}{*}{$\mathrm{R}^{2}$} & \multirow[t]{2}{*}{$\mathrm{R}^{3}$} & \multicolumn{2}{|c|}{ Condition $\mathrm{A}^{\mathrm{a}}$} & \multicolumn{2}{|c|}{ Condition $\mathrm{B}^{\mathrm{b}}$} \\
\hline & & & & Reaction time (min) & Yield $^{\mathrm{c}}(\%)$ & Reaction time (min) & Yield ${ }^{\mathrm{c}}(\%)$ \\
\hline 1 & $\mathrm{Ph}$ & $\mathrm{H}$ & $-\mathrm{C}(\mathrm{O}) \mathrm{NH}_{2}$ & 20 & 80 & 1 & 82 \\
\hline 2 & 4- $\mathrm{MeC}_{6} \mathrm{H}_{4}$ & $\mathrm{H}$ & $-\mathrm{C}(\mathrm{O}) \mathrm{NH}_{2}^{2}$ & 20 & 88 & 1 & 85 \\
\hline 3 & $4-\mathrm{ClC}_{6} \mathrm{H}_{4}^{4}$ & $\mathrm{H}$ & $-\mathrm{C}(\mathrm{O}) \mathrm{NH}_{2}^{2}$ & 20 & 80 & 2 & 80 \\
\hline 4 & $2-\mathrm{EtOC}_{6} \mathrm{H}_{4}^{4}$ & $\mathrm{H}$ & $-\mathrm{C}(\mathrm{O}) \mathrm{NH}_{2}^{2}$ & 30 & 90 & 2 & 85 \\
\hline 5 & $4-\mathrm{EtOC}_{6} \mathrm{H}_{4}^{4}$ & $\mathrm{H}$ & $-\mathrm{C}(\mathrm{O}) \mathrm{NH}_{2}^{2}$ & 15 & 85 & 2 & 85 \\
\hline 6 & 1-Naphthyl & $\mathrm{H}$ & $-\mathrm{C}(\mathrm{O}) \mathrm{NH}_{2}^{2}$ & 25 & 78 & 3 & 75 \\
\hline 7 & $\mathrm{Ph}$ & $\mathrm{Ph}$ & $-\mathrm{C}(\mathrm{O}) \mathrm{NH}_{2}^{2}$ & 15 & 82 & 2 & 80 \\
\hline 8 & $4-\mathrm{ClC}_{6} \mathrm{H}_{4}$ & $\mathrm{Ph}$ & $-\mathrm{C}(\mathrm{O}) \mathrm{NH}_{2}^{2}$ & 20 & 85 & 3 & 90 \\
\hline 9 & $4-\mathrm{MeOC}_{6} \mathrm{H}_{4}$ & $\mathrm{Ph}$ & $-\mathrm{C}(\mathrm{O}) \mathrm{NH}_{2}^{2}$ & 30 & 82 & 2 & 80 \\
\hline 10 & $2-\mathrm{HOC}_{6} \mathrm{H}_{4}^{-}$ & $\mathrm{CH}_{3}$ & $-\mathrm{C}(\mathrm{O}) \mathrm{NH}_{2}^{2}$ & 20 & 92 & 2 & 85 \\
\hline 11 & $\mathrm{Ph}$ & $\mathrm{H}^{3}$ & 2,4-dinitrophenyl & 25 & 80 & 2 & 78 \\
\hline 12 & $4-\mathrm{MeC}_{6} \mathrm{H}_{4}$ & $\mathrm{H}$ & 2,4-dinitrophenyl & 15 & 85 & 2 & 90 \\
\hline 13 & 4- $\mathrm{ClC}_{6} \mathrm{H}_{4}^{4}$ & $\mathrm{H}$ & 2,4-dinitrophenyl & 20 & 78 & 2 & 82 \\
\hline 14 & $2-\mathrm{EtOC}_{6} \mathrm{H}_{4}^{4}$ & $\mathrm{H}$ & 2,4-dinitrophenyl & 35 & 82 & 3 & 85 \\
\hline 15 & $4-\mathrm{EtOC}_{6} \mathrm{H}_{4}^{4}$ & $\mathrm{H}$ & 2,4-dinitrophenyl & 20 & 90 & 1 & 87 \\
\hline 16 & 1-Naphthyl & $\mathrm{H}$ & 2,4-dinitrophenyl & 15 & 82 & 2 & 82 \\
\hline 17 & $\mathrm{Ph}$ & $\mathrm{Ph}$ & 2,4-dinitrophenyl & 30 & 85 & 1 & 80 \\
\hline 18 & $4-\mathrm{ClC}_{6} \mathrm{H}_{4}$ & $\mathrm{Ph}$ & 2,4-dinitrophenyl & 40 & 75 & 2 & 85 \\
\hline 19 & $4-\mathrm{MeOC}_{6} \mathrm{H}_{4}$ & $\mathrm{CH}_{3}$ & 2,4-dinitrophenyl & 35 & 80 & 2 & 92 \\
\hline 20 & $2-\mathrm{HOC}_{6} \mathrm{H}_{4}$ & $\mathrm{Ph}$ & 2,4-dinitrophenyl & 20 & 95 & 3 & 95 \\
\hline
\end{tabular}

ander classical heating. The best yields were obtained using THF and toluene as solvent for semicarbazones and phenylhydrazones, respectively. ${ }^{\mathrm{b}}$ Under microwave irradiation in solvent-free system. ${ }^{c}$ Yields refer to isolated product. 
toluene, ammonium chlorochromate supported onto alumina ( $2 \mathrm{mmol}$ ) was added. The reaction mixture was refluxed for the indicated times (Table 1). The progress of reaction was monitored by TLC. Upon completion of the reaction, the solvent was evaporated to dryness under reduced pressure and the residue was chromatographed over silica gel to yield the corresponding carbonyl compound (Table 1).

Regeneration of carbonyl compounds from semicarbazones and 2,4-dinitro-phenylhydrazones under microwave irradiation in solvent-free system

An appropriate either semicarbazone or 2,4dinitrophenylhydrazone ( $2 \mathrm{mmol})$ was thoroughly mixed with the above catalyst $(2 \mathrm{mmol})$ in a beaker, using a spatula. An exothermic reaction ensues with darkening of the reagent. The beaker was placed in a house hold microwave oven and irradiated. The progress of reaction was monitored by TLC. Upon the completion of reaction, the product was extracted with $\mathrm{CH}_{2} \mathrm{Cl}_{2}(2 \times 5 \mathrm{~mL})$ and passed through a small bed of silica gel to afford the pure carbonyl compound (Table 1).

\section{References}

1. Sander, S. R.; Karo, W.; Organic Functional Groups Preparation, $2^{\text {nd }}$ ed., Academic Press: London, 1989, Vol. 3, p. 431.

2. Ram, R. N.; Varsha, K.; Tetrahedron Lett. 1991, 32, 5829; Barhate, N. B.; Gajare, A. S.; Wakharkar, R. D.; Sudlai, A.; Tetrahedron Lett. 1997, 38, 653.

3. Bahar, S.; Guha, S.; Synth. Commun. 2005, 35, 1183; Das, N. B.; Nayak, A.; Nanda, B.; J. Chem. Res. 2004, 10, 712; De, S. K.; Synth. Commun. 2004, 34, 2751; De, S. K.; Lett. Org. Chem. 2005, 2, 77; Ganguly, N. C.; Datta, M.; De, P.; J. Indian Chem. Soc. 2004, 81, 308; De, S. K.; Tetrahedron Lett. 2003, 44, 9055; Khaleghi, Sh.; Heravi, M. M.; Fotouhi, L. ; Phosphorus Sulfur Silicon Relat. Elem. 2003, 178, 155; Srinivas, K. V. N. S.; Das, N. B.; J. Chem. Res. 2002, 110, 556; Das, N. B.; Nanda, B.; Najak, A.; Synth. Commun. 2002, 32, 3647; Aghapoor, K.; Heravi, M. M.; Nooshabadi, M. A.; Ghassemzadeh, M. Monatsh. Chem. 2002, 133, 107; Heravi, M. M.; Ajami, D.; Mohajerani, B.; Ghassemzadeh, M.; Monatsh. Chem. 2001, 132, 881; Heravi, M. M.; Tajbakhsh, M.; Bakooie, H.; Ajami, D.; Monatsh. Chem. 1999, 130, 933.
4. Kiasat, A. R.; Kazemi, F.; Nourbakhsh, K.; Phosphorus Sulfur Silicon Relat. Elem. 2004, 179, 569; Karchaudhuri, N.; De, A.; Mitra, A. K. ; Indian J. Chem. 2003, 42B, 1537; Heravi, M. M.; Tajbakhsh, M.; Habibzadeh, S.; Ghassemzadeh, M.; Phosphorus Sulfur Silicon Relat. Elem. 2002, 177, 2299.

5. Zhang, G. S.; Chai, B.; Indian J. Chem. 2001, 40B, 1264; Heravi, M. M.; Hekmatshoar, R.; Beheshtiha, Y. S.; Ghassemzadeh, M.; Monatsh. Chem. 2001, 132, 651; Heravi, M. M.; Kiakojoori, R.; Mojtahedi, M. M.; Indian J. Chem. 2001, 40B, 329; Heravi, M. M.; Beheshtiha, Y. S.; Oskooie, S. H. A.; Ghassemzadeh, M.; Monatsh. Chem. 2000, 131, 187; Heravi, M. M.; Mirza-Aghayan, M.; Z. Naturforsch. 1999, 54B, 815; Heravi, M. M.; Kiakojoori, R.; Tabar-Hydar, K.; Monatsh. Chem. 1999, 130, 581; Heravi, M. M.; Kiakojoori, R.; TabarHydar, K.; J. Chem. Res. 1998, 656; Zhang, G. S.; Shi, O. Z.; Chen, M. F.; Cai, K.; Synth. Commun. 1997, 27, 953.

6. Gendre, F.; Yang, M.; Diaz, P.; Org. Lett. 2005, 7, 2719; Donati, D.; Morelli, C.; Taddei, M.; Tetrahedron Lett. 2005, 46, 2817; Desai, A.; Danks, T. N.; Wagner, G.; Tetrahedron Lett. 2005, 46, 955; Heravi, M. M.; Hekmatshoar, R.; Pedram, L.; J. Mol. Catal. A: Chem. 2005, 231, 89; Heravi, M. M.; Ajami, D.; Ghassemzadeh, M.; Synthesis 1999, 393; Heravi, M. M.; Ajami, D.; Aghapoor, K.; Ghassemzadeh, M.; Chem. Commun. 1999, 833.

7. For recent review on microwave-assisted chemical reactions, see Hayes, B. L.; Aldrichimica Acta 2004, 37, 66 and references cited therein; Kappe, C. O.; Angew. Chem. Int. Ed. 2004, 43, 6250; Majdoub, M.; Loupy, A.; roudesli, M. S.; Tetrahedron 1996, 52, 617; Kingston, H. M.; Jassie, L. B; Introduction to Microwave Sample Preparation, ACS: Washington, DC, 1988, ch. 2 .

8. Blackburn, O.; Tetrahedron Lett. 2005, 46, 1405 and references cited therein.

9. Agarwal, A.; Chauhan, P. M. S.; Tetrahedron Lett. 2005, 46, 1345; Heravi, M. M.; Nami, N.; Oskooie, S. H. A.; Hekmatshoar, R.; Phosphorus Sulfur Silicon Relat. Elem. 2005, 180, 1605; Heravi, M. M.; Kazemian, P.; Oskooie, S. H. A.; Ghassemzadeh, M.; J. Chem. Res. 2005, 2, 105.

10. Heravi, M. M.; Ajami, D.; Ghassemzadeh, M.; Tabar-Hydar, K.; Synth. Commun. 2001, 31, 2097.

11. Heravi, M. M.; Ajami, D.; Aghapoor, K.; Ghassemzadeh, M.; Chem. Commun. 1999, 833; Heravi, M. M.; Ajami, D.; Mohajerani, B.; Tabar-Heydar, K.; Ghassemzadeh, M.; Synth. Commun. 2002, 32, 3325; Mohajerani, B.; Heravi, M. M.; Ajami, D.; Monatsh. Chem. 2001, 132, 871.

Received: September 3, 2005 Published on the web: March 3, 2006 